\title{
An Intelligent Broadcasting Algorithm for Early Warning Message Dissemination in VANETs
}

\author{
Ihn-Han Bae \\ School of IT Engineering, Catholic University of Daegu, Gyeongsan 712-702, Republic of Korea \\ Correspondence should be addressed to Ihn-Han Bae; ihbae@cu.ac.kr
}

Received 2 January 2014; Revised 14 October 2014; Accepted 14 October 2014

Academic Editor: Valentina Emilia Balas

Copyright (C) 2015 Ihn-Han Bae. This is an open access article distributed under the Creative Commons Attribution License, which permits unrestricted use, distribution, and reproduction in any medium, provided the original work is properly cited.

\begin{abstract}
Vehicular ad hoc network (VANET) has gained much attention recently to improve road safety, reduce traffic congestion, and enable efficient traffic management because of its many important applications in transportation. In this paper, an early warning intelligence broadcasting algorithm is proposed, EW-ICAST, to disseminate a safety message for VANETs. The proposed EW-ICAST uses not only the early warning system on the basis of time to collision (TTC) but also the intelligent broadcasting algorithm on the basis of fuzzy logic. Thus, the EW-ICAST resolves effectively broadcast storm problem and meets time-critical requirement. The performance of EW-ICAST is evaluated through simulation and compared with that of other alert message dissemination algorithms. From the simulation results, we know that EW-ICAST is superior to Simple, P-persistence, and EDB algorithms.
\end{abstract}

\section{Introduction}

VANETs have been considered as an important communication infrastructure for the intelligent transportation systems (ITS). In IEEE 802.11p, the dedicated short range communication (DSRC) is a core function and it is a US government project for vehicular network communication for the enhancement of driving safety and comfort of automotive drivers. DSRC-based communication devices are expected to be installed in the future [1].

VANETs raise new challenges to the design of data communication protocols due to the high dynamicity of the underlying topology, the intermittent connectivity, and fast changing density. Broadcasting is the message delivery task from a source node to all other nodes in a network to enhance the safety of drivers and provide the comfortable driving environment. Many important VANET services, ranging from safety applications to location-based advertisement, rely on the reliability and efficiency of underlying broadcast protocols. Applications have different requirements on broadcast protocol design. Location-based advertisement emphasizes reliability in order to achieve higher coverage of vehicles, while warning delivery, which broadcasts emergent information to approaching vehicles, requires both low propagation delay and reliability. Because of the shared wireless medium, blindly broadcasting packets may lead to frequent contention and collisions among transmitting neighboring nodes. This problem is sometimes referred to as the broadcast storm problem. While multiple solutions exist to alleviate the effects of the broadcast storm problem in MANET, only a few solutions have been proposed to resolve this issue in VANET $[2,3]$.

The main contribution of this paper is to present EWICAST, an early warning intelligence broadcasting algorithm for safety message dissemination in VANET. The proposed EW-ICAST uses not only the early warning system on the basis of TTC but also the intelligent broadcasting algorithm on the basis of fuzzy logic. In EW-ICAST, when a driving vehicle recognizes that the brake light of a vehicle right ahead is on or a vehicle of lateral lane is lane changing through vehicle-to-vehicle ( $\mathrm{V} 2 \mathrm{~V})$ communication, the vehicle computes TTC. If the TTC was less than or equal to a threshold of TTC, the vehicle broadcasts an alert message to following vehicles. Then a vehicle receives an alert message for the first time, and the vehicle determines rebroadcast degree using fuzzy logic rules, where the rebroadcast degree depends on the current traffic density of road and the distance between previous hop vehicle and current receiving vehicle. 
The probability of rebroadcasting the message, as well as the rebroadcast delay, is dependent on the computed rebroadcast degree. If the vehicle did not receive the rebroadcasted alert message from another vehicle until a time-out delay expires, the vehicle rebroadcasts the alert message with the rebroadcast probability.

The remainder of this paper is organized as follows. Section 2 reviews related work. Section 3 describes the proposed EW-ICAST algorithm. Section 4 offers a performance evaluation of EW-ICAST through simulation. Finally, Section 5 concludes the paper and discusses directions for future investigations.

\section{Related Work}

2.1. VANET Routing Mechanism. VANET routing mechanism is classified into four broad categories: unicast, multicast, geocast, and broadcast approaches $[4,5]$. Unicastrouting is a fundamental operation for vehicle to construct a sourceto-destination routing in a VANET. Multicastis defined by delivering multicast packets from a single source vehicle to all multicast members by multihop communication. Geocastrouting is to deliver a geocast packet to a specific geographic region. Vehicles located in this specific geographic region should receive and forward the geocast packet; otherwise, the packet is dropped. Broadcastprotocol is utilized for a source vehicle sending broadcast message to all other vehicles in the network as shown.

2.2. Broadcast Protocols for VANET. The primary goal for safety alert application is to deliver the alert message to all vehicles approaching the incident site, so that drivers may be alerted prior to their natural visual reaction. So end-toend delay for the alert message has to be minimized. The previous systems for alert message broadcast dissemination in VANETs are divided into two groups, one where vehicles are not equipped with GPS and the other where they are equipped with GPS.

(1) GPS Not Equipped. Simple broadcast $[6,7]$ is the simplest protocol used in V2V safety alert application for VANET in the literal sense of the words. When there is an accident, safety alert application will send alert messages to all vehicles approaching towards accident site. When a vehicle receives a broadcast message for the first time, it retransmits the message. The vehicle ignores all subsequent broadcast messages it receives from other vehicles rebroadcasting the same message.

There are two main problems in this simple broadcast method. First, there are many redundant rebroadcast messages because of flooding. Thus, when $n$ hosts receive the message for the first time, $n$ replications will result and there is a high chance that the message will be received by many hosts located in a close proximity.

P-persistence [7, 8] tries to reduce the broadcast storm problem by using a stochastic selection strategy to decide the vehicles that will rebroadcast the alert message. When a vehicle receives a broadcast message for the first time, the vehicle will rebroadcast the alert message with a random probability $P$. This method will help reduce the number of rebroadcasting vehicles and alleviate the effects of the broadcast storm. However failures to extend the alert message decide not to, which will cause the loss of alert message.

(2) GPS Equipped. Unlike the P-persistence or gossip-based scheme, weighted $P$-persistence [3] assigns higher probability to nodes that are located farther away from the broadcaster given that GPS information is available and accessible from the packet header.

Upon receiving a packet from node $i$, node $j$ checks the packet ID and rebroadcasts with probability $P_{i j}$ if it received the packet for the first time; otherwise, it discards the packet. Denoting the relative distance between nodes $i$ and $j$ by $D_{i j}$ and the average transmission range by $R$, the forwarding probability, $P_{i j}$, can be calculated on a per packet basis using the following simple equation:

$$
P_{i j}=\frac{D_{i j}}{R} .
$$

Li et al. [9] proposed a novel broadcast protocol called efficient directional broadcast (EDB) for urban VANET using directional antennas. Due to the fact that the topology of VANET changed rapidly, EDB makes receiver-based decisions to forward the packet with the help of the GPS information. The receiver only needs to forward the packet in the opposite direction where the packet arrives. After a vehicle receives a packet successfully, it waits for a time before taking a decision whether to forward the packet or not. During this time, the vehicle listens to other relays of the same packet. The waiting time can be calculated using the following equation:

$$
\text { Waiting Time }=\left(1-\frac{D}{R}\right) \times \operatorname{maxWT} \text {, }
$$

where $D$ is the distance from the sender which can be obtained using the sender's location information added in the packet and its own and $R$ is the transmission range. The maxWT is a configurable parameter which can be adjusted according to the density of the vehicle.

2.3. Collision Warning Systems. This section describes the models proposed for the collision warning system based on sensor information and for driver evasive action in response to collision warnings issued by the system. In multilane road environment, vehicles move in both longitudinal and lateral directions. Accordingly, vehicles can be expected to cause both longitudinal collisions and lateral collisions. Therefore, the collision warning system model is conceived of as encompassing two subsystems: a forward vehicle collision warning system to address longitudinal collisions and a side collision warning system to address lateral collisions as shown in Figure 1 [10].

(1) Forward Vehicle Collision Warning. Figure 2 illustrates the variables used in this paper. The current time is set to zero. The position, velocity, and acceleration of the preceding 


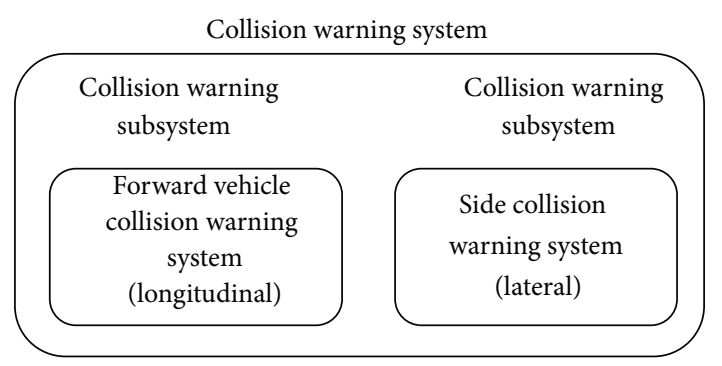

FIGURE 1: Structure of a collision warning system.

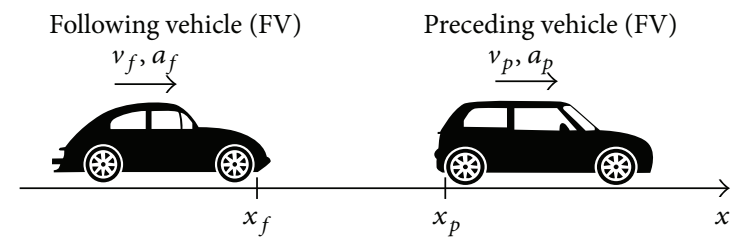

FIGURE 2: Definition of variables. $x$ : position $[\mathrm{m}], v$ : velocity $[\mathrm{m} / \mathrm{s}]$, $a$ : acceleration $\left[\mathrm{m} / \mathrm{s}^{2}\right], d_{f}\left(=-a_{f}\right)$ : deceleration of $\mathrm{FV}\left[\mathrm{m} / \mathrm{s}^{2}\right]$, and $T$ : reaction time $[\mathrm{s}]$.

vehicle at that time are defined as $x_{p_{0}}, v_{p_{0}}, a_{p_{0}}$, and the position, velocity, and acceleration of the following vehicle are defined as $x_{f_{0}}, v_{f_{0}}, a_{f_{0}}$. Furthermore, the relative position, relative velocity, and relative acceleration are defined as $x_{r_{0}}=$ $x_{f_{o}}-x_{p_{0}}, v_{r_{0}}=v_{f_{0}}-v_{p_{0}}$, and $a_{r_{0}}=a_{f_{0}}-a_{p_{0}}$.

Assume that the preceding vehicle suddenly decelerates and that the following vehicle then decelerates after the reaction time $T$; the intervehicular distance $d$ when the two vehicles stop can be described as [11]

$$
d=-x_{r}-v_{f} \cdot T+\left(\frac{v_{f}^{2}}{2 a_{f}}-\frac{v_{p}^{2}}{2 a_{p}}\right) .
$$

The condition of forward collision warning is satisfied when the following distance obtained by the sensors $D$ becomes smaller than the calculated intervehicular distance $d$, called the stopping.

(2) Side Collision Warning. Sensors are capable of detecting both distance to and speed of (both laterally and longitudinally for each) all vehicles with radius $R[\mathrm{~m}]$ and viewing angle $\pm \phi\left[{ }^{\circ}\right]$. Based on the accumulated data of every refresh cycle, the system decides to issue a warning when warning assessment criteria are satisfied both laterally and longitudinally [10]. The formula for the warning assessment criteria is defined as follows:

$$
x_{r} \geq v_{r} \times T \text {. }
$$

(3) Time to Collision. One of the most representative indices for assessing the warning provision timing of the forward obstacle collision warning system (FOCWS) is TTC [11]. The TTC is defined as follows:

$$
\mathrm{TTC}=-\frac{x_{r}}{v_{r}}=\frac{x_{f}-x_{p}}{v_{f}-v_{p}} .
$$

The TTC represents the predicted time to collision on the assumption that the current relative velocity is maintained.

In the situation in which the adjacent vehicle avoids the collision by applying the brakes when the subject vehicle changes lanes, the following conditions are required to prevent the adjacent vehicle from colliding with the preceding vehicle in the lane change, which means that the headway distance before lane changing must be less than the necessary distance for the following vehicle's deceleration [12]:

$$
\begin{gathered}
v_{r} \cdot \mathrm{TTC}>v_{r} \cdot T+\frac{v_{r}^{2}}{2 \alpha}, \\
\text { TTC }>T+\frac{v_{r}}{2 \alpha},
\end{gathered}
$$

where $\alpha$ represents the following vehicle's deceleration.

When it is assumed that $T=1$ second, $v_{r}=30 \mathrm{Km} / \mathrm{h}$, and $\alpha=4 \mathrm{~m} / \mathrm{s}^{2}$, TTC required to avoid the collision is calculated to be over 2.04 seconds. Braking alone will not avoid the collision when TTC is less than 2 seconds.

\section{EW-ICAST Design}

In this paper, we present EW-ICAST to improve the propagation of traffic safety application in VANET. In the design of EW-ICAST, we assume the following:

(i) before transmitting an alert message, the on-board GPS is used to calculate the distance between the previous hop vehicle and the current receiving vehicle;

(ii) further, the GPS is used to calculate the speed of the current receiving vehicle;

(iii) all vehicles are equipped with multiple directional antennas that are the antennas which radiate greater power in one or more directions allowing for increased performance on transmitting and receiving and reduced interference from unwanted sources.

The proposed EW-ICAST uses not only the early warning system on the basis of TTC but also the intelligent broadcasting algorithm on the basis of fuzzy logic. In EW-ICAST, when a driving vehicle recognizes that a vehicle right ahead steps on the brakes or a vehicle of lateral lane changes lanes through $\mathrm{V} 2 \mathrm{~V}$ communication, the vehicle computes TTC. If the TTC was less than or equal to a threshold value of TTC, the vehicle broadcasts an alert message to following vehicles. When a vehicle receives an alert message for the first time, if the current speed of the vehicle was higher than a threshold value of vehicle speed, HI-CAST (hybrid intelligent broadcast) is performed. Otherwise, I-CAST (intelligent broadcast) is performed. The structure of EW-ICAST algorithm is shown in Figure 3.

In I-CAST, the receiving vehicle determines rebroadcast degree using fuzzy logic rules, where the rebroadcast degree depends on the current traffic density of road and the distance between previous hop vehicle and current receiving vehicle. The probability of rebroadcasting the message, as well as the rebroadcast delay, is dependent on the computed rebroadcast degree. If the vehicle did not receive the rebroadcasted alert 


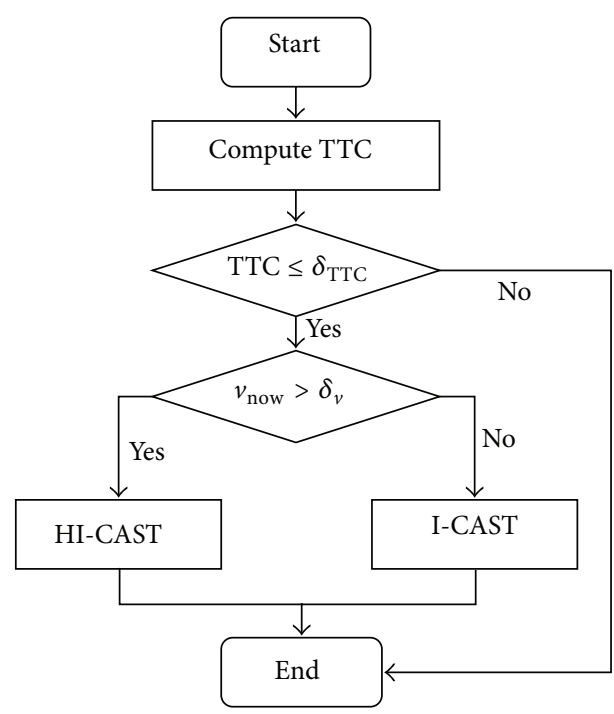

FIgURE 3: Structure of EW-ICAST algorithm.

message from another vehicle until a time-out delay expires, the vehicle rebroadcasts the alert message with the rebroadcast probability. HI-CAST uses I-CAST in conjunction with alert token protocols, RPB-TOKEN, where RPB- (relative position based-) TOKEN sends an alert token to the neighboring vehicle in opposite direction.

We map the speed of the current receiving vehicle $(v)$ to five basic fuzzy sets, VF (very fast), F (fast), M (medium), $\mathrm{S}$ (slow), and VS (very slow), using the fuzzy function as shown in Figure 4. The membership function of $v$ represents fuzzy set of $v$. The membership function which represents a fuzzy set of $v$ is denoted by $\mu_{V D}(v)$, where $V$ represents the maximum speed of vehicles.

Figure 5 shows a few examples of EW-ICAST, where S0, S1, S2, S3, and S4 represent the segments that divide the transmission range into the equal-size blocks, respectively. S0 and S4 represent the nearest and the farthest segments from a collision warning point, respectively. Firstly, consider the I-CAST scenario in Figure 5(a) where vehicles exist in the transmission range. Vehicle A which detects the collision warning broadcasts an alert message to all vehicles in its transmission range. Vehicle D travelling in $\mathrm{S} 4$ has very short waiting time, but vehicle B which is in S2 has long waiting time. If the current speed of vehicle $\mathrm{D}$ was medium, vehicle $\mathrm{D}$ has high rebroadcast probability. Vehicle $\mathrm{D}$ rebroadcasts with high probability if the vehicle $\mathrm{D}$ received the alert message for the first time and has not received any duplicates before its waiting time; otherwise, it discards the alert message. Secondly, consider the HI-CAST scenario depicted in Figure 5(b) where no vehicles exist within transmission range. If the current traffic density of the road was very low or the current speed of receiving vehicle is very fast, the RPB-TOKEN sends an alert token to the neighboring vehicle in opposite direction. Vehicle $\mathrm{B}$ receives the alert token from vehicle $\mathrm{A}$ which detects collision warning and then sends the alert token to vehicle $C$ travelling straight head. Vehicle $\mathrm{C}$ sends the alert token to vehicle $\mathrm{D}$ travelling

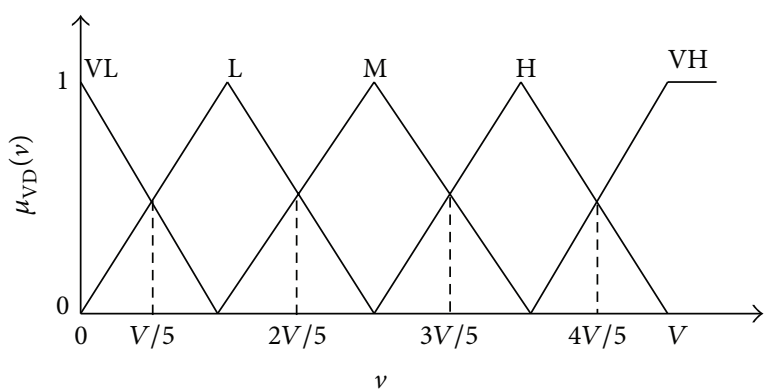

FIGURE 4: Membership function for the current speed.

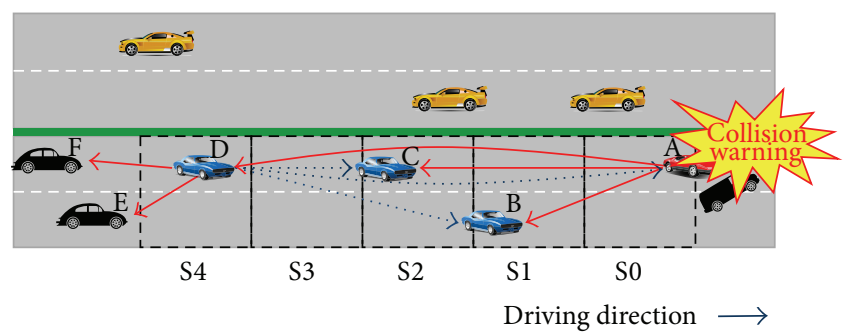

(a) I-CAST scenario

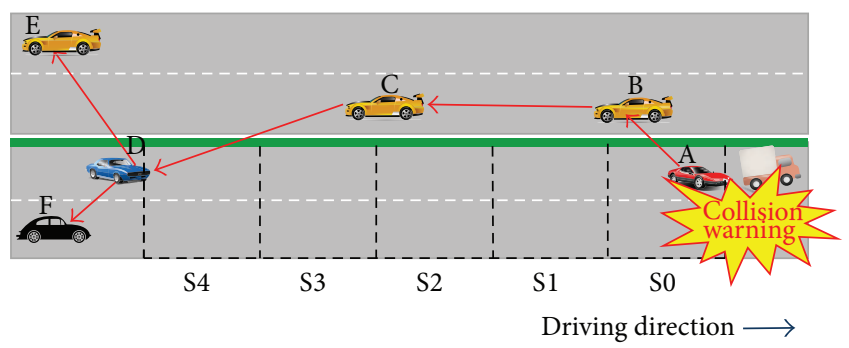

(b) HI-CAST scenario

FIGURE 5: Illustrating the working of EW-ICAST.

in opposite direction. Vehicle D broadcasts the alert message to all vehicles in transmission range, and the vehicle sends the alert token to neighboring vehicle $\mathrm{E}$ in opposite direction.

For I-CAST, the control rules of rebroadcast degree which consider the current speed of receiving vehicle and the distance between previous hop vehicle and receiving vehicle are shown in Table 1.

Upon receiving an alert message from vehicle $i$, vehicle $j$ calculates Rebroadcast $_{\text {Prob }}(i, j)$ and $\operatorname{segWT}(i, j)$ through $(7)$, where Rebroadcast Prob $(i, j)$ is the nonfuzzy control output and defuzzifier is the defuzzification operator [13]. Vehicle $j$ rebroadcasts with $\operatorname{Rebroadcast}_{\text {Prob }}(i, j)$ if vehicle $j$ received the alert message for the first time and has not received any duplicates before $\operatorname{segWT}(i, j)$; otherwise, it discards the alert message. Consider

$$
\begin{array}{r}
\operatorname{Rebroadcast}_{\text {Prob }}(i, j) \\
=\text { defuzzifier }_{\left(\begin{array}{c}
\text { a linguistic weighted } \\
\text { factor for rebroadcasting }
\end{array}\right),} \\
\text { where } 0 \leq \text { Rebroadcast }_{\text {Prob }}(i, j) \leq 1,
\end{array}
$$


TABLE 1: The control rules for rebroadcast degree.

\begin{tabular}{lccccc}
\hline Segment & & \multicolumn{3}{c}{ VD } \\
& VS & S & M & F & VF \\
\hline S0 & VL & VL & L & L & M \\
S1 & VL & L & L & M & M \\
S2 & L & L & M & M & H \\
S3 & L & M & M & H & VH \\
S4 & M & M & H & VH & VH \\
\hline
\end{tabular}

Notes: (input variables) VD: VF, very fast; F, fast; $\mathrm{M}$, medium; S, slow; VS, very slow.

(Output variables) rebroadcast degree: $\mathrm{VH}$, very high; $\mathrm{H}$, high; $\mathrm{M}$, medium; L, low; VL, very low.

$$
\begin{gathered}
\text { defuzzifier } \left.\left(\begin{array}{c}
\mathrm{VH} \\
\mathrm{H} \\
\mathrm{M} \\
\mathrm{L} \\
\mathrm{VL}
\end{array}\right\}\right)=\left\{\begin{array}{l}
1.0 \\
0.8 \\
0.6 \\
0.4 \\
0.2
\end{array}\right\}, \\
\operatorname{segWT}(i, j)=\left(1-\frac{\mathrm{SN}(j)}{N}\right) \times \operatorname{maxsegWT},
\end{gathered}
$$$$
\text { where } 0 \leq \operatorname{segWT}(i, j) \leq \operatorname{maxsegWT} \text {. }
$$

And $\mathrm{SN}(j)$ represents segment number in which the current receiving vehicle $j$ is travelling, $N$ is the largest number of segments, and maxsegWT represents the maximum segment waiting time which is determined by considering the number of segments and the transmission delay of a VANET.

We also propose an alert token passing algorithm on the basis of RPB-MACn [14] that is called RPB-TOKEN. Figure 6 depicts such a directional antenna enabled MAC design based on the run-time static relative position property in VANETs. Here, all vehicles are equipped with 8 statically configured directional antennas, each dedicated to one relative position vicinity, operating over the single wireless channel to communicate with its 1-hop neighbors, and $T n$ indicates the transmission over wireless channel $n$, while $R n$ indicates the reception over channel $n$.

When a traffic accident has occurred, if the current traffic density of road was low, the vehicle that detects the accident initiates RPB-TOKEN. RPB-TOKEN sends an alert token with initial hop count value to a neighboring vehicle in the opposite direction. Upon receiving the token, the vehicle sends the alert token to the vehicle ahead of it on the road. The next receiving vehicle sends the alert token to the vehicle traveling in opposite direction. If the vehicle next after received the alert message for the first time, the receiving vehicle broadcasts the alert message to all vehicles in transmission range. And the receiving vehicle sends the alert token with initial hop count value to the neighboring vehicle in opposite direction.

\section{Performance Evaluation}

The primary objective of EW-ICAST is to improve the success rate of safety message dissemination, that is, the percentage of

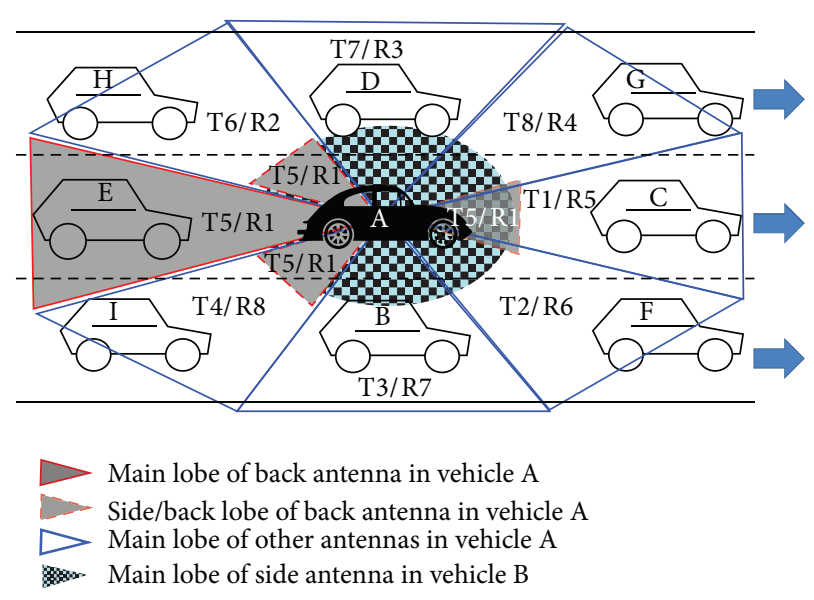

FIgURE 6: Directional antenna with dedicated channel pair.

vehicles that receive the safety alert message. EW-ICAST also aims to mitigate the effect of the broadcast storm problem that afflicts most of the VANET's safety alert protocols. Three metrics are used to evaluate different protocols.

(i) Collision: the number of alert message collisions that occur during the period of simulation.

(ii) Success rate: percentage of vehicles that received alert message.

(iii) Time: time delay from accident that occurred until last vehicle received alert message.

The parameters and values of the performance evaluation for EW-ICAST are shown in Table 2, where the alert region represents the circular area within which the message is transmitted and PHY/MAC layers are compliant with IEEE 802.11p draft standard [15]. The current speed of vehicles depends on the traffic density of the road. Thus, the higher the traffic density, the lower the vehicle speed; similarly, the lower the traffic density, the faster the vehicle speed. Accordingly, the current speed of a vehicle is computed from the following equation:

$$
v_{\text {now }}=v_{\max } \times\left(1-\frac{\rho_{\text {now }}}{\rho_{\text {max }}}\right),
$$

where $v_{\max }$ represents the maximum allowable speed of the road, $\rho_{\max }$ represents the traffic density in which the vehicle speed is zero when a traffic jam occurred, and $\rho_{\text {now }}$ represents the current traffic density of the road.

The performance of EW-ICAST is evaluated by using MATLAB 7.0 [16], where the dataset of traffic density follows Gaussian distribution, and the evaluation results are derived from the simulation program running of 5 times a case.

Figure 7 shows average number of alert messages that are transmitted by RPB-TOKEN accordingly to traffic densities in case the radius of alert region is $10 \mathrm{Km}$. The threshold value of traffic density is the traffic density that may have no vehicles in transmission range. Thus, the threshold value of traffic density is determined through the simulation result of Figure 7 and (8). 
TABLE 2: Simulation parameters.

\begin{tabular}{lc}
\hline Parameter & Value \\
\hline Radius of alert region & $2 \sim 10 \mathrm{Km}$ \\
Transmission range $(R)$ & $500 \mathrm{~m}$ \\
The length of a segment & $100 \mathrm{~m}$ \\
Deceleration $(\alpha)$ & $4.0 \mathrm{~m} / \mathrm{s}^{2}$ \\
Reaction time $(T)$ & $1 \mathrm{sec}$ \\
Threshold value of TTC $\left(\delta_{\text {TTC }}\right)$ & $2 \mathrm{sec}$ \\
Threshold value of vehicle speed $\left(\delta_{V}\right)$ & $75 \mathrm{Km} / \mathrm{h}$ \\
Traffic density (TD) & $20 \sim 140 \mathrm{vehicles} / \mathrm{Km}$ \\
Maximum traffic density (maxTD) & $160 \mathrm{vehicles} / \mathrm{Km}$ \\
Traffic deviation & $(1.5 *$ maxTD $) / \mathrm{TD}$ \\
Maximum speed of vehicles & $100 \mathrm{Km} / \mathrm{h}$ \\
Initial hop count value & 2 \\
Number of lanes & 4 \\
The broadcast probability in $P$-persistence & 0.5 \\
Transmission delay & $20 \mathrm{~ms} / \mathrm{hop}$ \\
Maximum waiting time & $120 \mathrm{~ms}$ \\
Maximum segment waiting time & $110 \mathrm{~ms}$ \\
\hline
\end{tabular}

Figure 8(a) shows the number of alert message collisions that has occurred accordingly to the radius of alert region in case traffic density is low. While the number of collisions of EW-ICAST is smaller than that of Simple and P-persistence algorithms, the number of collisions of EW-ICAST is approximately equal to that of EDB algorithm. Figure 8(b) shows, respectively, numbers of alert message collisions for EWICAST and EDB that have occurred accordingly to the radius of alert region in case traffic density is high, where the performance of EW-ICAST is extremely better than that of EDB because EW-ICAST uses the fuzzy control rules for rebroadcast degree that consider the speed of a receiving vehicle and the distance segment between the previous hop vehicle and the receiving vehicle.

The most important result, the success rate for different algorithms in case traffic density is 30 vehicles per kilometer, is shown in Figure 9. The loss of alert message causes low success rate. The success rate of EW-ICAST is higher than that of Simple and P-persistence algorithms, and the success rate of EW-ICAST is equal to that of EDB algorithm which achieves perfect success rate through broadcasting an alert message every 10 maxWT until a next hop neighbor appears.

Message dissemination delay in case traffic density is 30 vehicles per kilometer is shown in Figure 10, where the network transmission time for alert message is only considered, but the delay time of PHY/MAC layers due to alert messages congestion and collision is not considered. EW-ICAST uses TTC based early collision warning system. The vehicle that expects a collision broadcasts an alert message to all following vehicles in advance before the vehicle collision occurs. The delay time of EW-ICAST algorithm is very much shorter than that of other algorithms, while the delay time of EDB has the worst delay time because multiple maxWT delays are continued until a next hop neighbor appears.

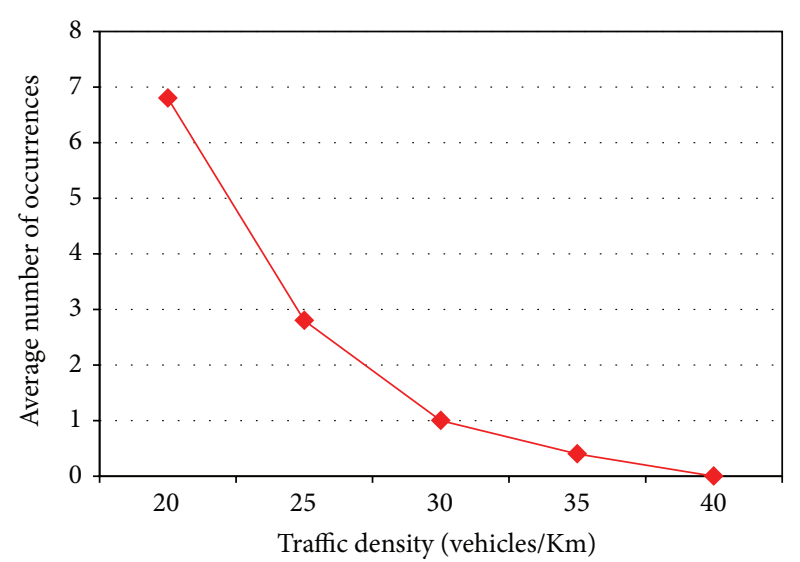

FIGURE 7: Average number of transmitted alert messages with RPBTOKEN.

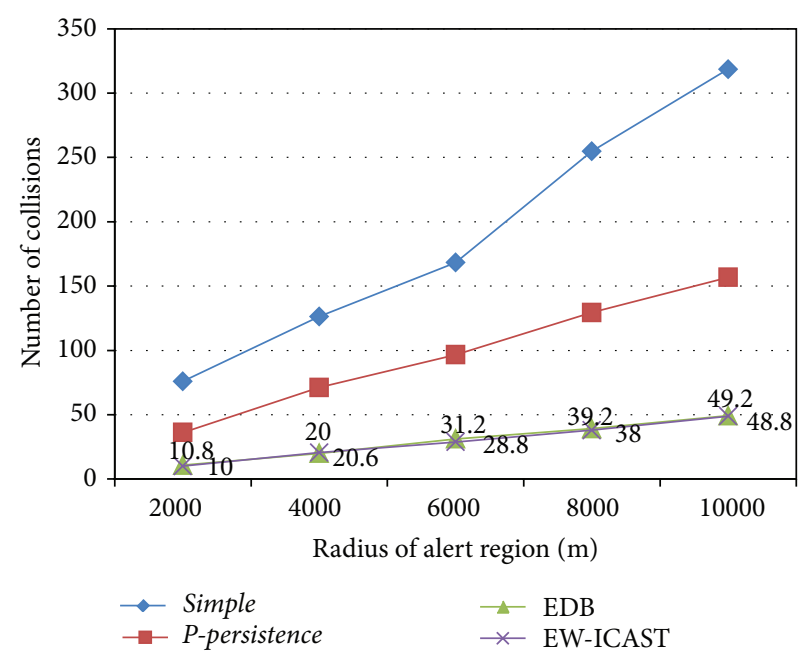

(a) $\mathrm{TD}=30$ vehicles $/ \mathrm{Km}$

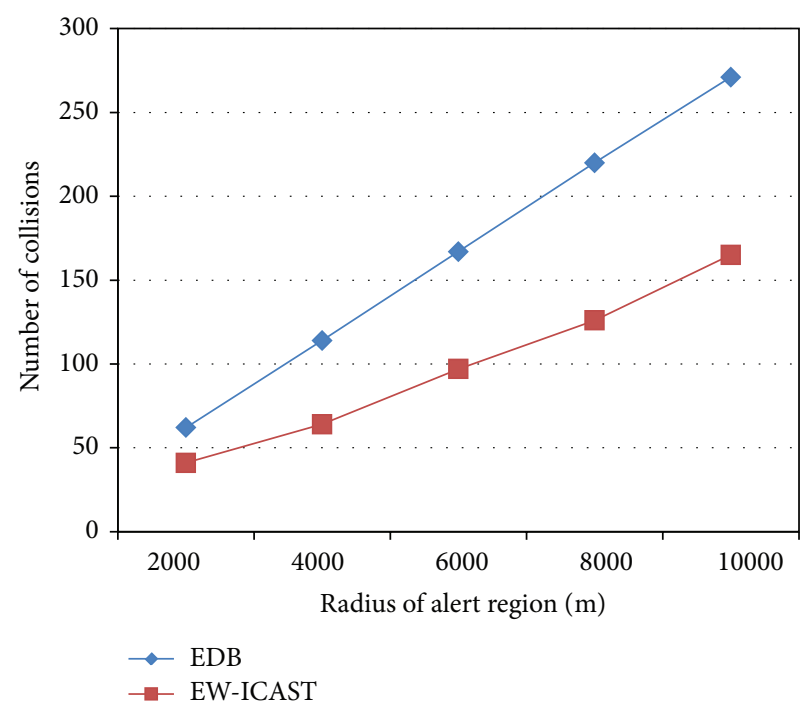

(b) $\mathrm{TD}=140$ vehicles $/ \mathrm{Km}$

FIGURE 8: Number of collisions with alert region radius. 


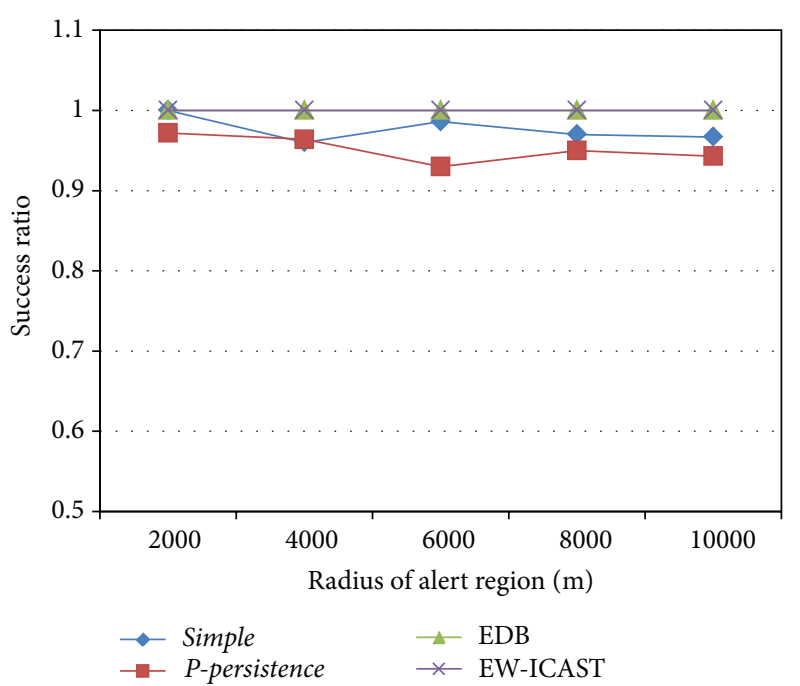

FIGURE 9: Success rate with alert region radius.

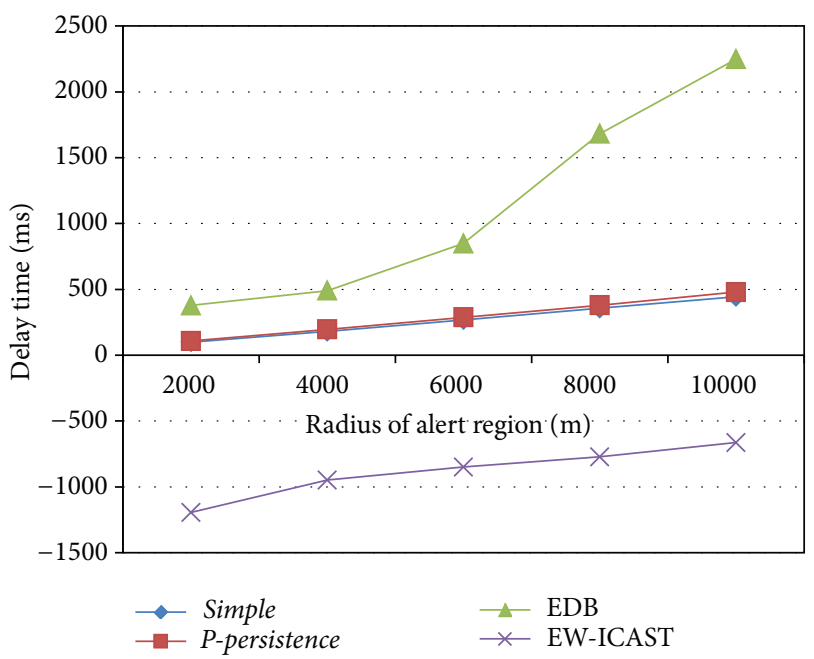

FIGURE 10: Delay time with alert region radius.

Figure 11 shows the number of occurrences of the fuzzy sets for rebroadcast in EW-ICAST in three cases in which the current traffic density is 40,90 , and 140 vehicles per kilometer, respectively, where the radius of alert region is $10 \mathrm{Km}$. The fuzzy set in which rebroadcast probability is very high $(\mathrm{VH})$ occurred more frequently in case traffic density is low (TD = 40). But the fuzzy set in which rebroadcast probability is medium (M) only occurred in case traffic density is high $(\mathrm{TD}=140)$.

\section{Conclusion}

Most VANET applications favor broadcast transmission that addresses the broadcast storm problem to avoid unnecessary loss of information during dissemination. Emergency warning for public safety is one of the many applications that are highly time-critical and require more intelligent broadcast mechanism than just blind flooding.

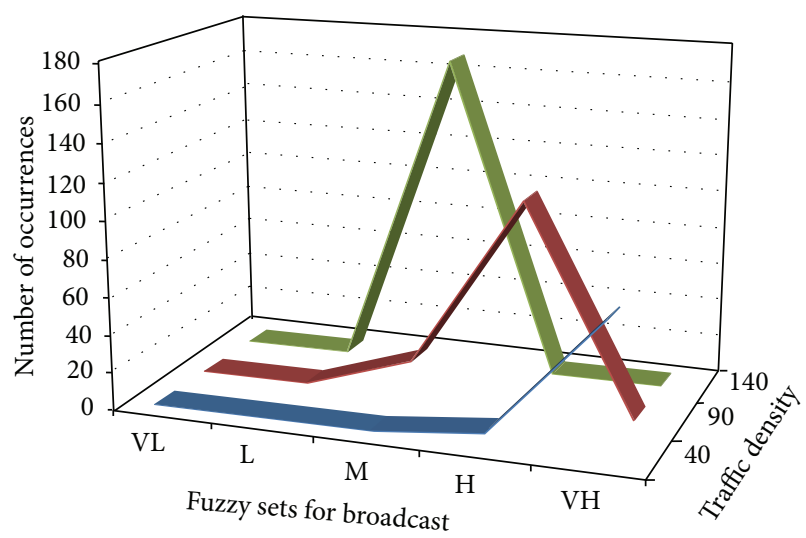

FIGURE 11: Number of occurrences for fuzzy sets in EW-ICAST.

In this paper, we proposed EW-ICAST which used not only the early warning system on the basis of TTC but also the intelligent broadcasting algorithm on the basis of fuzzy logic. The performance of EW-ICAST was evaluated through simulation and compared with that of other alert message dissemination algorithms. From the simulation results, we knew that the EW-ICAST was superior to Simple, P-persistence, and EDB algorithms. Therefore, the EW-ICAST resolved effectively broadcast storm problem and met time-critical requirement. Our future work includes studying an adaptive alert message dissemination algorithm which considers road conditions and shapes.

\section{Conflict of Interests}

The author declares that there is no conflict of interests regarding the publication of this paper.

\section{References}

[1] M. Chitra and S. S. Sathya, "Efficient broadcasting mechanisms for data dissemination in vehicular ad hoc networks," International Journal of Mobile Network Communications \& Telematics, vol. 3, no. 3, pp. 47-63, 2013.

[2] J. Liu, Z. Yang, and I. Stojmenovic, "Receiver consensus: ontime warning delivery for vehicular ad-hoc networks," IEEE Transaction on Emerging Topics in Computing, vol. 1, no. 1, pp. 57-68, 2013.

[3] N. Wisitpongphan, O. K. Tonguz, J. S. Parikh, P. Mudalige, F. Bai, and V. Sadekar, "Broadcast storm mitigation techniques in vehicular ad hoc networks," IEEE Wireless Communications, vol. 14, no. 6, pp. 84-94, 2007.

[4] E. Schoch, F. Kargl, and M. Weber, "Communication patterns in VANETs," IEEE Communications Magazine, vol. 46, no. 11, pp. $119-125,2008$.

[5] Y.-W. Lin, Y.-S. Chen, and S.-L. Lee, "Routing protocols in vehicular Ad Hoc networks: a survey and future perspectives," Journal of Information Science and Engineering, vol. 26, no. 3, pp. 913-932, 2010.

[6] O. Tonguz, N. Wisitpongphan, F. Bai, P. Mudalige, and V. Sadekar, "Broadcasting in VANET," in Proceedings of the Mobile 
Networking for Vehicular Environments (MOVE '07), pp. 7-12, Anchorage, Alaska, USA, May 2007.

[7] K. Suriyapaibonwattana and C. Pomavalai, "An effective safety alert broadcast algorithm for VANET," in Proceedings of the International Symposium on Communications and Information Technologies (ISCIT '08), pp. 247-250, Vientiano, Laos, October 2008.

[8] K. Suriyapaiboonwattana, C. Pornavalai, and G. Chakraborty, "An adaptive alert message dissemination protocol for VANET to improve road safety," in Proceedings of the IEEE International Conference on Fuzzy Systems, pp. 1639-1644, Jeju Island, Republic of Korea, August 2009.

[9] D. Li, H. Huang, X. Li, M. Li, and F. Tang, "A distancebased directional broadcast protocol for urban vehicular ad hoc network," in Proceedings of the International Conference on Wireless Communications, Networking and Mobile Computing (WiCOM '07), pp. 1520-1523, Shanghai, China, September 2007.

[10] Y. Takatori and T. Hasegawa, "Stand-alone collision warning systems based on information from on-board sensors," IATSS Research, vol. 30, no. 6, pp. 39-47, 2006.

[11] T. Hiraoka, M. Tanaka, H. Kumamoto, T. Izumi, and K. Hatanaka, "Collision risk evaluation index based on deceleration for collision avoidance (first report)," Review of Automotive Engineering, vol. 30, no. 4, pp. 429-437, 2009.

[12] T. Wakasugi, "A study on warning timing for lane decision aid systems based on driver's lane change Maneuver," in Proceedings of the International Technical Conference on the Enhanced Safety of Vehicles, pp. 1-7, Washington, DC, USA, June 2005.

[13] C. C. Lee, "Fuzzy logic in control systems: fuzzy logic controller. I," IEEE Transactions on Systems, Man, and Cybernetics, vol. 20, no. 2, pp. 404-418, 1990.

[14] C. Chigan, V. Oberoi, and J. Li, "RPB-MACn: a relative position based collision-free MAC nucleus for vehicular ad hoc networks," in Proceedings of the IEEE Global Telecommunications Conference, pp. 1-6, San Francisco, Calif, USA, December 2006.

[15] Y. Toor, P. Mühlethaler, A. Laouiti, and A. de La Fortelle, "Vehicle ad hoc networks: applications and related technical issues," IEEE Communications Surveys and Tutorials, vol. 10, no. 3, pp. 74-88, 2008.

[16] M. G. Kay, Basic Concepts in Matlab, 2010, http://www.ise.ncsu .edu/kay/Basic_Concepts_in_Matlab.pdf. 


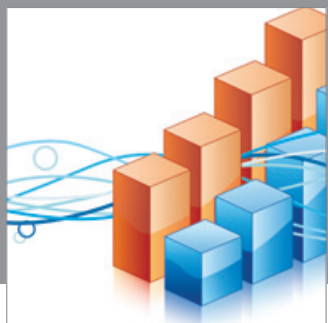

Advances in

Operations Research

mansans

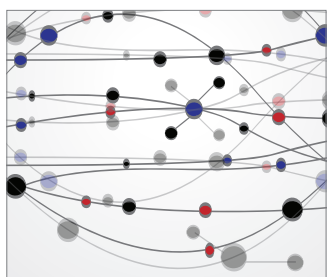

The Scientific World Journal
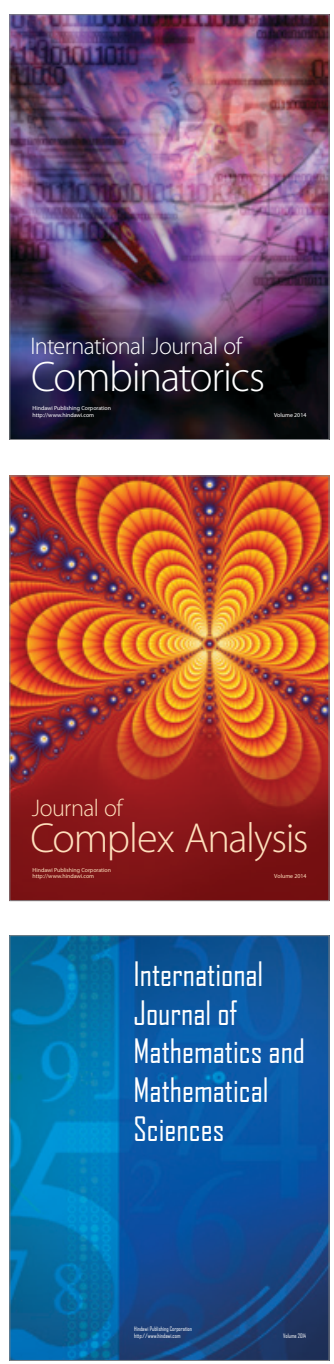
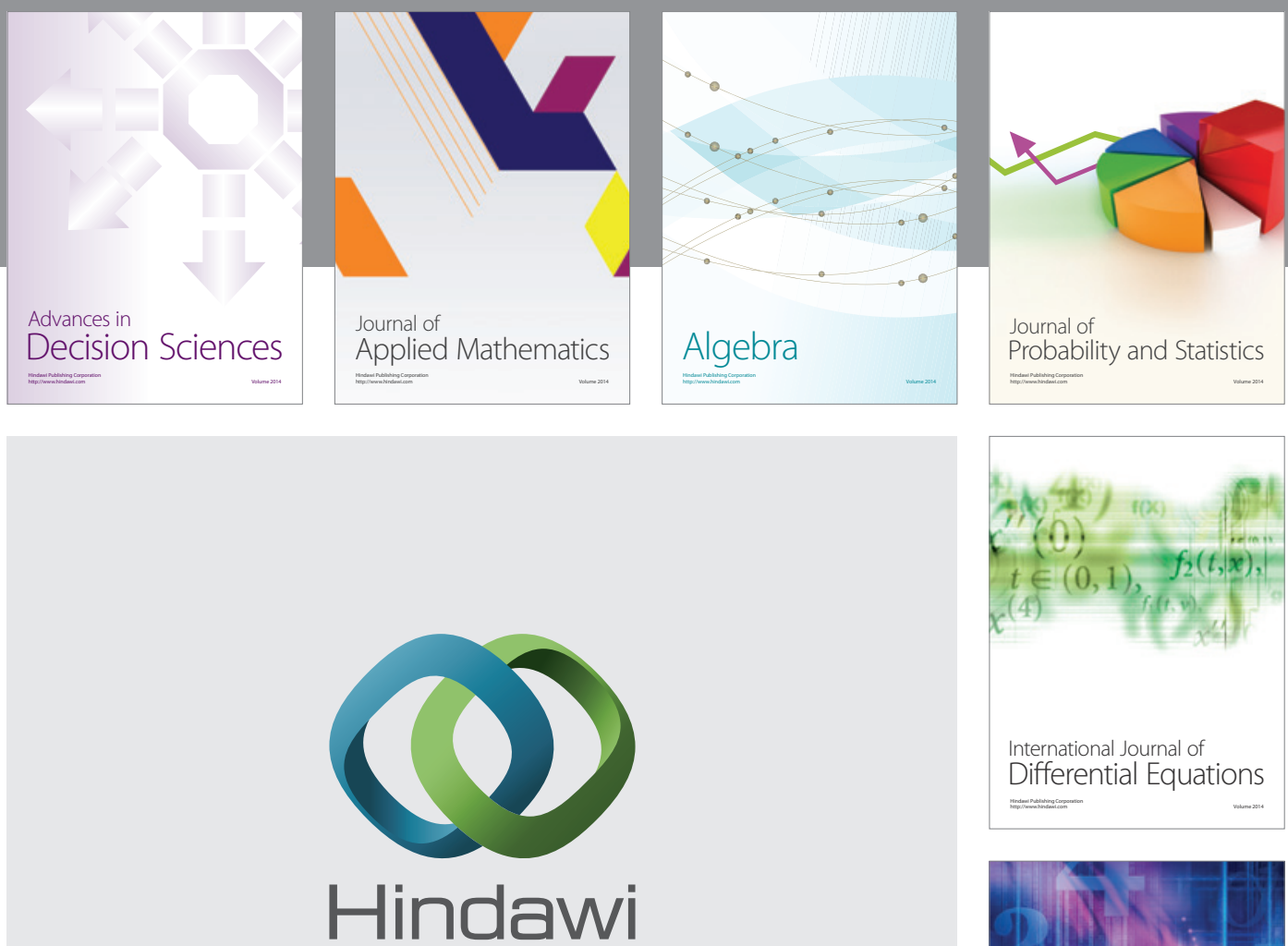

Submit your manuscripts at http://www.hindawi.com
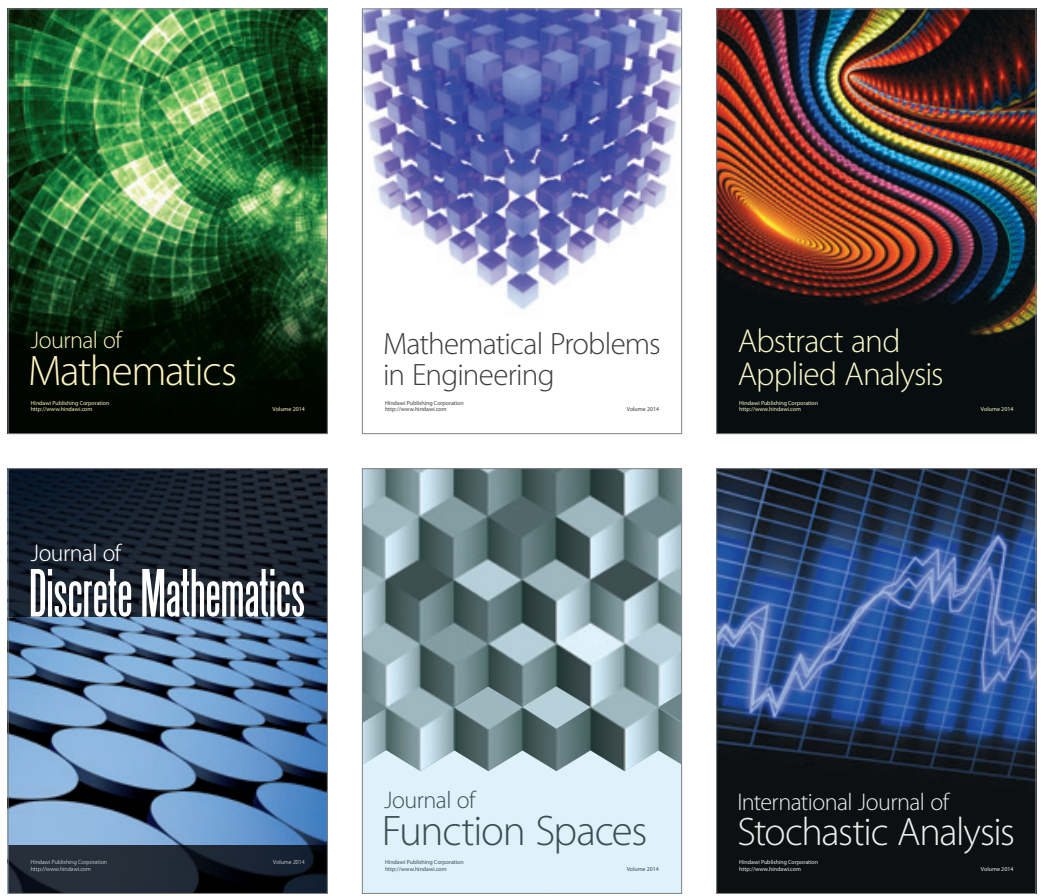

Journal of

Function Spaces

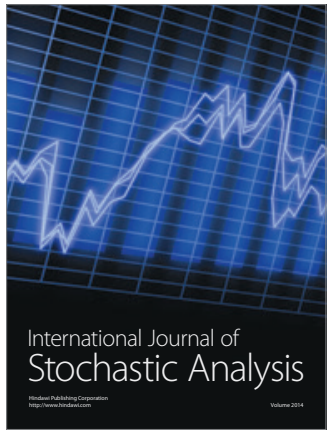

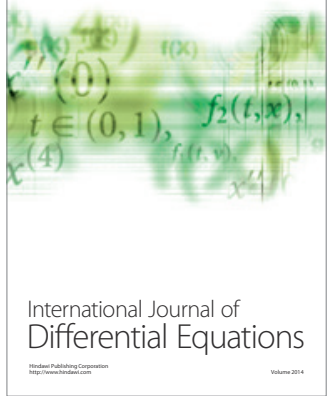
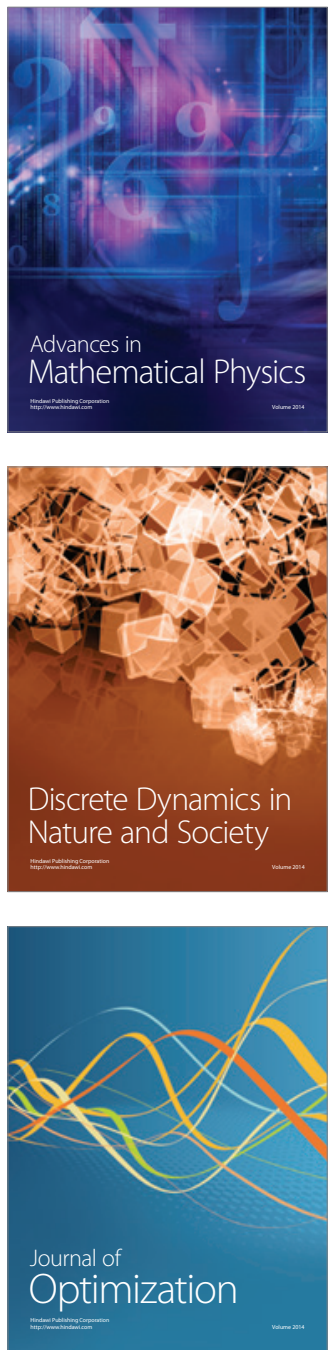